Musées, Patrimoine et Culture scientifiques et techniques

$116 \mid 2008$

mars - avril 2008

\title{
Phæno, un centre de sciences futuriste à la frontière entre art et science
}

\section{(2) OpenEdition}

Journals

Édition électronique

URL : http://journals.openedition.org/ocim/373

DOI : $10.4000 /$ ocim.373

ISSN : 2108-646X

Éditeur

OCIM

\section{Édition imprimée}

Date de publication : 1 mars 2008

Pagination : 28-35

ISSN : 0994-1908

\section{Référence électronique}

«Phæno, un centre de sciences futuriste à la frontière entre art et science », La Lettre de I'OCIM [En

ligne], 116 | 2008, mis en ligne le 01 janvier 2011, consulté le 19 avril 2019. URL : http:// journals.openedition.org/ocim/373 ; DOI : 10.4000/ocim.373 
Aujourd'hui, Wolfsburg reste le site central du groupe Volkswagen avec 50000 employés. Depuis quelques années, la ville s'attache à changer son image de ville industrielle dortoir et à améliorer sa qualité de vie grâce à la construction de nouvelles infrastructures culturelles et de loisirs.

En 2000 a été inaugurée la Autostadt de Volkswagen, une sorte de parc d'attractions dans lequel les clients viennent chercher leur nouvelle voiture et passent leur journée en famille. La ville souhaitait donner une réponse forte à cette inauguration. Le responsable des affaires culturelles de l'époque, Wolfgang Guthardt aujourd'hui directeur du Phæno - lança alors l'idée d'un grand projet d'infrastructure culturelle. Les deux raisons principales qui lui donnèrent liidée de réaliser un centre de sciences sont la visite du Technorama en Suisse et parallèlement la lecture d'un article sur les science centers aux États-Unis paru dans le quotidien allemand Frankfurter Allgemeine Zeitung (édition du 27 juin 1998). Larticle est consacré au succès rencontré par le nouveau California Science Center à Los Angeles ; il dresse un historique de l'apparition des sciences centers aux États-Unis en s'attardant notamment sur le rôle de précurseur joué par l'Exploratorium de San Francisco, fondé par Frank Oppenheimer à la fin des années 1960. Il décrit en outre la philosophie des sciences centers : amener les jeunes visiteurs aux sciences en présentant des expériences surprenantes, vivantes, ludiques, émotionnelles et sensorielles, moins basées sur une approche intellectuelle et le «pathos muséal ».

Il ne restait plus dès lors qu'à convaincre le maire et le conseil municipal de construire un centre de sciences à Wolfsburg. Une décision somme toute naturelle dans cette ville tellement liée à l'industrie automobile, qui n'a pas d'autres racines et qui ne possède pas d'université. Ce projet apparaît ainsi adapté aux décideurs politiques. L'objectif assigné à Phæno est d'œuvrer à la diffusion des sciences et technologies auprès du grand public, de captiver les jeunes générations et de les motiver pour les sciences et les techniques : une ambition importante pour le site central du groupe Volkswagen et au regard du manque actuel d'effectifs dans les filières scientifiques.

La ville a financé l'ensemble du projet (bâtiment et exposition) à hauteur de 80 millions d'euros. Phæno est situé à l'extrémité nord de Wolsburg, au cœur des voies de communication permettant d'accéder à la ville, à côté de la gare ICE (nom du TGV allemand) et en face de la Autostadt.

\section{Phæno, un monument de l'architecture moderne}

La jeune ville de Wolfsburg a déjà fait appel par le passé à des architectes de renom pour la réalisation de plusieurs de ses bâtiments publics; citons par exemple Hans Scharoun pour le théâtre municipal ou bien Alvar Aalto pour la maison de la culture. Dans la veine de cette tradition, c'est l'architecte Zaha Hadid que le jury a sélectionné pour la réalisation du Phæno.

Zaha Hadid est la première femme à avoir reçu en 2004 le prix Pritzker, qui fait office de prix Nobel des architectes. Longtemps ses projets ont eu la réputation de ne pas être réalisables. Mais depuis peu, en partie grâce à la réalisation du Phæno, ils voient le jour dans le monde entier, comme l'Aquatic Center prévu pour les Jeux Olympiques de Londres en 2012. Le choix s'est donc porté sur une architecte qui travaille à des projets très expérimentaux et non conventionnels, essayant sans relâche de découvrir de nouvelles formes. À travers ce choix pour un projet avant-gardiste, la ville de Wolfsburg reste en outre dans la continuité de son « Leitsatz » (programme d'orientation) intitulé " Lust an Entdeckungen » (Envie de découvertes), initié il y a quelques années.

Zaha Hadid a dessiné le bâtiment du Phæno et ses abords comme une forme fluide où topographies extérieure et intérieure sont étroitement liées, une gigantesque sculpture de béton, d'acier et de verre,

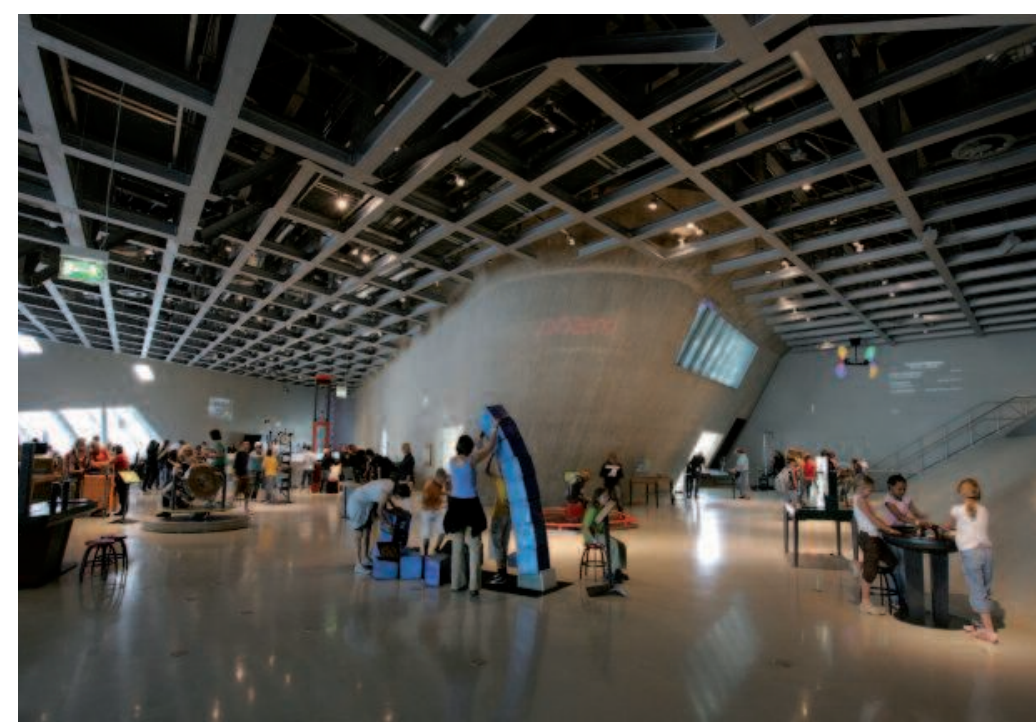

Disposition intérieure des éléments d'exposition interactifs () Volkswagen 
que l'on peut parcourir dehors et dedans. Certains voient dans le bâtiment un bateau à vapeur, d'autres un reptile, ou un iceberg, la plupart cependant un ovni ou un vaisseau spatial. Le bâtiment mesure 150 métres de long et 16 mètres de haut. La surface de l'exposition est portée par 10 cônes à géométrie variée. On peut ainsi se déplacer sous la surface d'exposition, au milieu de ces cônes. Ce paysage pourrait avoir été sculpté par l'érosion de courants fluviaux.

Les cônes abritent pour la plupart des espaces tels que l'entrée et les caisses, la restauration, les ateliers, la boutique, le théâtre, le forum des idées (un espace réservé à des workshops et à des expositions). Cinq de ces cônes traversent la surface d'exposition. Une imposante structure autoportante, faite de poutres métalliques, repose sur ces cinq cônes et supporte le toit.

L'architecture spectaculaire du Phæno a suscité un important écho médiatique qui démontre, s'il en était besoin, le rôle primordial que joue actuellement l'architecture pour la promotion dans les médias des projets culturels. En octobre 2007, le journal britannique The Guardian a sélectionné dans un cycle «Great modern buildings » Phæno parmi les 12 plus importants bâtiments de l'architecture moderne à côté par exemple du musée Guggenheim à Bilbao, de l'Empire State Building à New York et de l'Opéra de Sydney ${ }^{(1)}$.

\section{Une exposition de type "Science Center »: I'influence de l'Exploratorium}

L'exposition du Phæno s'inspire donc fortement des expériences menées à l'Exploratorium qui offrent au visiteur la possibilité d'expérimenter, de découvrir par lui-même, de jouer. Sur une surface intérieure de $9000 \mathrm{~m}^{2}$ que l'on peut comparer à un paysage ouvert formé de plateaux, de cratères, de cavernes, de collines sont disposées environ 250 stations d'expérimentation. Il n'y a pas de scénographie d'exposition à proprement parler : les stations sont installées " nues » dans ce paysage, sans ajout d'éléments scénographiques qui pourraient concurrencer l'architecture ou interférer avec elle de façon négative. Ainsi, les phénomènes scientifiques sont placés au premier plan. Ce sont eux qui par leur esthétique, leur côté spectaculaire, doivent fasciner les visiteurs : une part importante du temps et du financement a donc été consacrée à la conception et à la production de ces stations d'expérimentation.

La philosophie de Phæno est «Ich entdecke ! » (Je découvre!). Le visiteur découvre de son propre chef

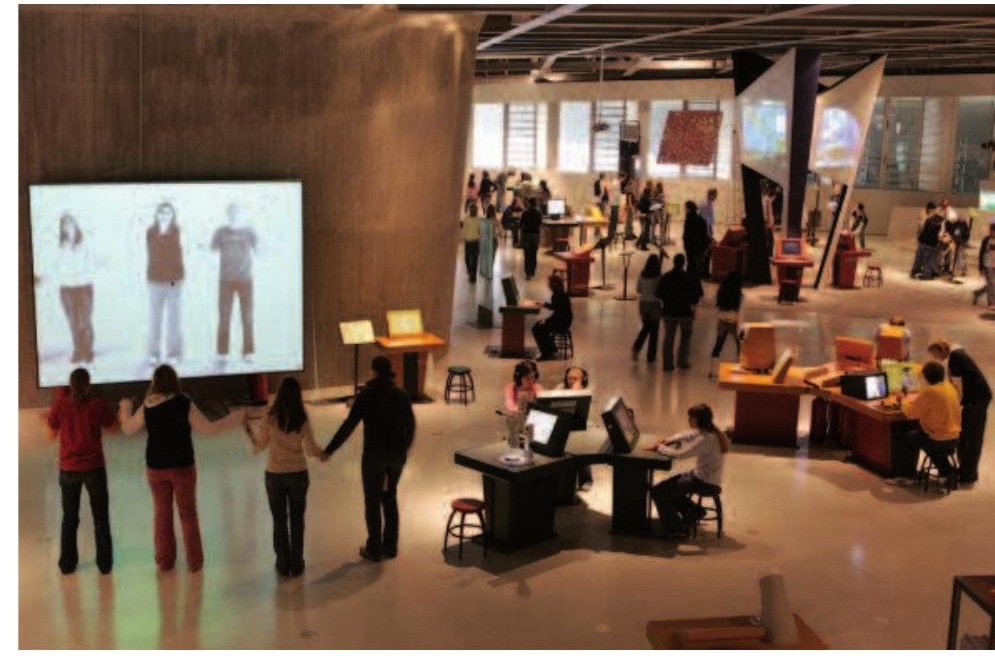

Une scénographie dépouillée (C) Phæno

ce " paysage d'expérimentation ", décide librement de ses déplacements. Il n'y a pas de chemin de visite pré-programmé, pas de sections thématiques séparées les unes des autres. Les stations ne sont pas pour autant disposées aléatoirement : beaucoup d'entre elles sont placées de façon à se compléter thématiquement. Cela ne signifie pas que le visiteur est laissé seul dans l'exposition. À ses côtés se trouvent les «phænomen » et « phænowomen », le personnel employé dans l'exposition, qui joue un rôle primordial au niveau de l'accompagnement de l'exposition et du service par rapport aux publics. Là encore, cette philosophie vient des pays anglo-saxons. On peut la résumer par «Be a gift to the spirit » (être un cadeau à l'esprit), c'est-àdire qu'il ne s'agit pas - tel un enseignant - de transmettre des savoirs au public, mais plutôt d'être un accompagnateur qui « découvre » en même temps que le visiteur et l'inspire, l'incite à découvrir, expérimenter et jouer par lui-même.

Le commissaire de l'exposition, Joe Ansel, est américain. Il a exercé diverses responsabilités à l'Exploratorium de San Francisco. Encadré par un jury d'experts, il a sélectionné les 250 stations originales et les a fait construire par environ 20 entreprises réparties dans le monde entier, apportant beaucoup d'attention à leur design et à leur esthétique (2).

La grande particularité de Phæno est la présence parmi ces stations d'œuvres d'artistes qui se trouvent en quelque sorte à la frontière entre arts et sciences, entre arts et techniques. Ce choix s'inscrit à nouveau dans la continuité de l'Exploratorium, « The museum 
of science, art and human perception » et l'héritage intellectuel de Frank Oppenheimer, qui souhaitait œuvrer au rapprochement entres arts et sciences et considérait d'ailleurs les deux comme appartenant à une seule culture. Ces artistes sont plutôt des bricoleurs de génie, des "Géo-trouve-tout ", qui pour beaucoup d'entre eux ont travaillé en tant qu'artistes en résidence à l'Exploratorium et bénéficient donc d'une expérience de plusieurs années à ce niveau.

Ces installations utilisent des phénomènes scientifiques, des constructions techniques pour produire une émotion chez le spectateur, le surprendre, l'amuser... Ces stations ont une fonction primordiale dans le Phæno en liant l'œuvre d'art qu'est l'architecture et l'exposition, et en permettant de toucher un large public qui au départ n'est pas nécessairement intéressé par les sciences et techniques. Sur les 40 installations artistiques, 20 ont été développées spécialement pour le Phæno (3). Comme pour toutes les stations de l'exposition, il y a un texte décrivant ce que le visiteur doit faire, ce qu'il peut observer et le phénomène scientifique démontré : certaines des stations proposent un texte plus complet qui permet d'approfondir les informations sur le phénomène scientifique observé.

Nous n'avons pas encore réalisé d'études complètes sur la réception de ces stations par le public, mais les premières observations laissent apparaitre qu'elles font partie des secteurs les plus appréciés de l'exposition permanente. Il arrive souvent que des visiteurs, a fortiori réticents à l'idée de visiter une exposition traitant de sciences ou bien venus avant tout pour voir l'architecture du bâtiment, soient agréablement surpris et même subjugués par ces installations. Il faut cependant faire remarquer que le public ne fait pas forcément la distinction entre ces installations et les autres, car elles sont parfaitement intégrées dans l'exposition générale, la seule différence notable étant la mention du nom de l'artiste dans le texte explicatif de l'installation.

\section{Une large polette d'activités}

Parallèlement à l'exposition permanente, Phæno propose aux publics (individuels et groupes) un certain nombre d'activités interactives, parfois temporaires - dans le TechLab, les visiteurs ont la possibilité de participer à des travaux manuels de " bricolage » et de construction, comme par exemple la fabrication d'instruments de musiques réalisés à partir de matériaux du quotidien ; - dans le BioLab les visiteurs peuvent faire des expériences de biologie ou de chimie.

Les expériences-énigmes sont une autre forme intéressante d'interaction avec le public. Un phæno(wo)man réalise directement dans l'exposition de petites expériences scientifiques sous forme d'énigmes que les visiteurs doivent résoudre.

D'autres membres de l'équipe font des présentations appelées «Science Shows » dans lesquelles des

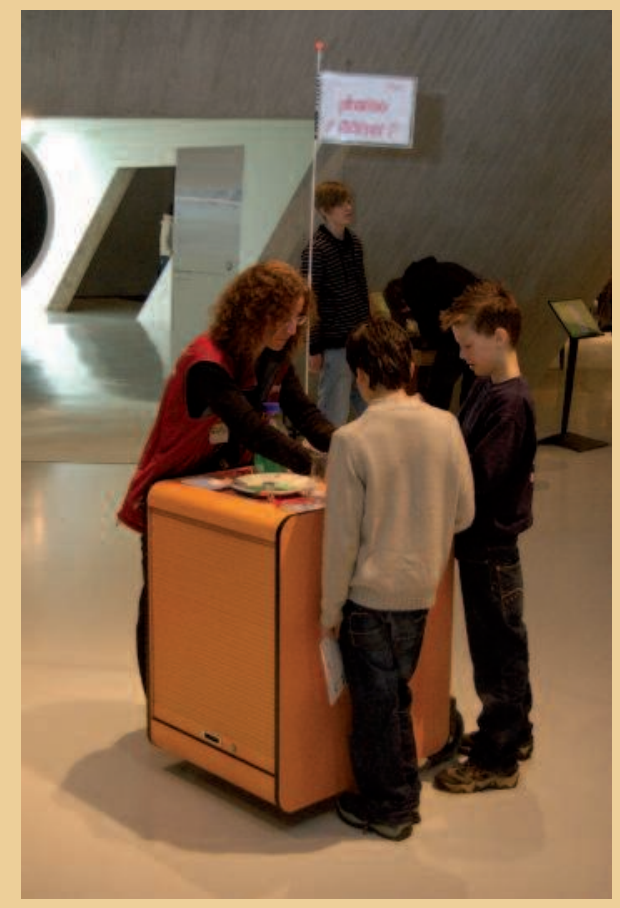

L'exposition permanente propose aux visiteurs un ensemble d'expériences-énigmes. (c) Phæno

(1)
réagir les spectateurs sur ce sujet d'éthique en les confrontant à une situation inattendue.

expériences scientifiques sont réalisées de manière humoristique et interactive. Ainsi, le « Gas Show » fait partie du programme depuis l'ouverture : on y montre des expériences sur les forces de l'air, des manipulations avec de la glace carbonique ou de l'azote liquide. Ces présentations ont lieu dans le Théâtre des Sciences (200 places), un espace séparé du reste de l'exposition.

Enfin, le centre de sciences propose d'autres formats, plus ponctuels comme ce projet de théâtre interactif, " Le premier homme cloné », dans lequel un comédien interprète le rôle fictif du premier homme cloné et discute sur le mode de l'improvisation avec le public, qui a la possibilité de lui poser des questions sur sa vie. Lobjectif de ce projet est d'envisager d'une manière volontairement polémique le sujet du clonage et de faire 


\section{Quelques exemples d'installations artistiques}

Afin de donner une idée plus précise des types d'installations proposées au public, nous présentons dans ce qui suit une sélection d'artistes et d'œuvres exposés au Phæno.

Nous commençons par deux artistes américains Arthur Ganson et Norman Tuck - qui sont présents dans l'exposition permanente mais également dans la première grande exposition temporaire au Phæno, PhantasieMechanik : maschinen erzählen Geschichten (Fantaisie Mécanique : des machines racontent des histoires) présentée jusqu'au 29 juin 2008 et qui montre des automates, machines et installations cinétiques.

Sculpteur de formation, Arthur Ganson se définit comme étant quelque part entre un artiste et un ingénieur, un inventeur et un poète. Depuis plus de 25 ans, il construit des sculptures cinétiques, qui sont littéralement de la «poésie en mouvement ». Les mouvements graciles de ses machines racontent des histoires, laissent réfléchir sur des aspects de l'existence humaine ou font tout simplement rire par leur côté souvent absurde. Ses travaux sont exposés aussi bien dans des musées d'art que de sciences. Ils couvrent une palette très large, allant de constructions massives en acier jusqu'à des figures filigranes en fil d'acier comportant des engrenages, que l'artiste fabrique lui-même. Ganson vit à proximité de Boston dans le Massachussetts. Il a été artiste en résidence à l'Exploratorium ou au MIT de Cambridge (États-Unis). Les mécanismes sont toujours visibles dans ses sculptures, révélant selon ses propres mots " les qualités humaines... la fragilité, la tristesse, la joie, la peur, et aussi l'étonnement ».

Deux œuvres d'Arthur Ganson sont exposées au Phæno : «The Little yellow chair » (La petite chaise jaune) et « Machine and concrete » (Machine et béton) :

- dans «The Little yellow chair », une étoile à 6 bras mécaniques se déploie et se recroqueville sans cesse. Au bout de chaque bras se trouve une pièce en bois jaune. Lorsque les bras se rapprochent, les 6 pièces se retrouvent pour une fraction de seconde au centre de l'étoile, formant une petite chaise jaune, avant de ré-éclater et de se séparer à nouveau, et ainsi de suite. Les 6 bras sont entraînés par une seule et même chaîne métallique, elle-même entrâ̂née par un moteur électrique. L'étoile déployée a un diamètre approximatif de $4 \mathrm{~m}$.
- dans «Machine and concrete », 25 engrenages sont installés en ligne les uns à côté des autres. Le mouvement de rotation d'un moteur électrique est transmis au premier engrenage qui transfert ce mouvement à l'engrenage suivant, et ainsi de suite... Le rapport de transmission est si faible (chacun des engrenages a 120 dents à l'extérieur et 14 dents à l'intérieur), que déjà à partir du quatrième engrenage, le mouvement de rotation n'est plus perceptible à l'œil nu. Le dernier engrenage est inséré dans un bloc de béton. Cette construction est possible grâce au jeu qui existe entre les différents engrenages. Il faudrait 594 billions d'années (1012) au dernier engrenage pour effectuer un tour complet !

Norman Tuck, également sculpteur de formation, vit et travaille à San Fransisco. C'est Joe Ansel qui l'introduit en 1985 dans le monde des musées de sciences. Il a également travaillé en tant qu'artiste en résidence à l'Exploratorium. Son exposition Art Machines a été montrée dans de nombreux musées de science d'Amérique du Nord. Tuck développe son art de façon à créer une expérience directe et ludique pour le spectateur. Ses installations s’inspirent de phénomènes

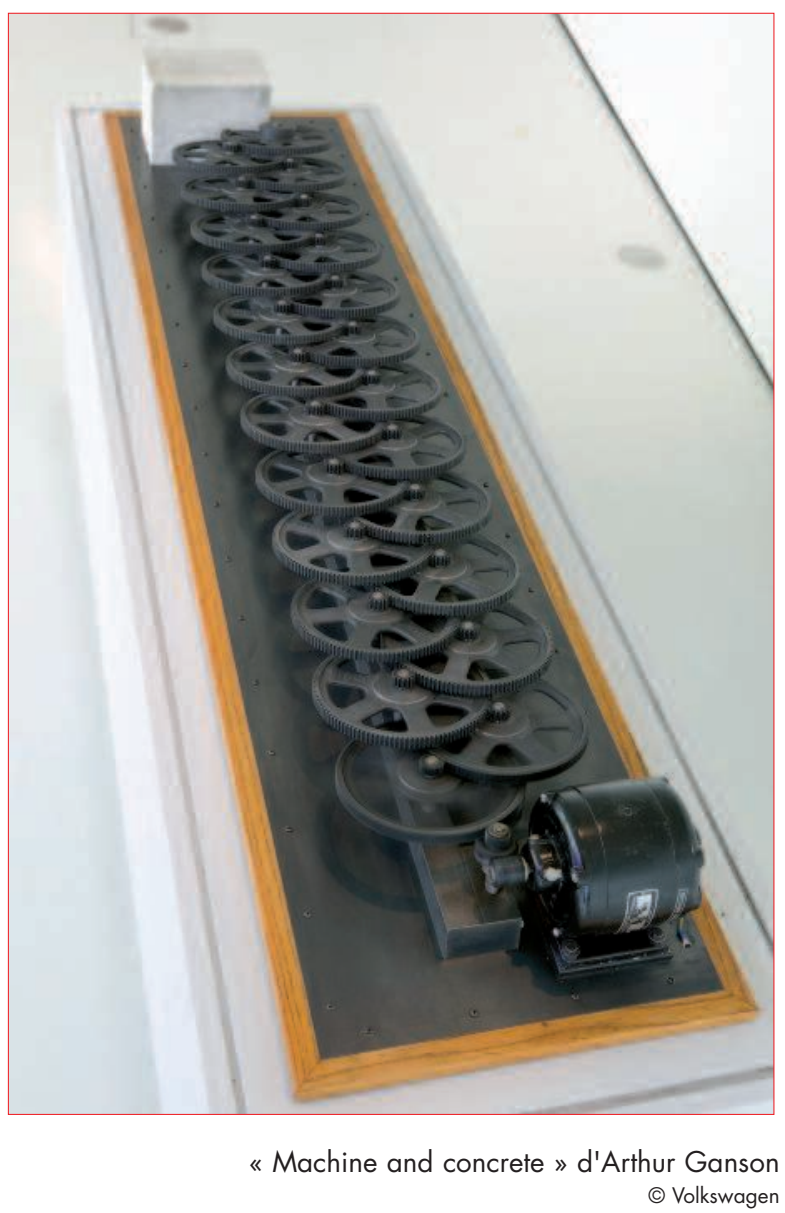


naturels tels que le mouvement d'ondes, la résonnance, la périodicité. En même temps, elles utilisent et transforment souvent des objets techniques du quotidien. Cinq installations de Norman Tuck sont exposées au Phæno : " Oscylinderscope », "Stepper organ », « Lariat Chain », « Three balls on a string » et « Disco ».

L'installation ingénieuse "Oscylinderscope »utilise des éléments de mécanique, optique et acoustique pour engendrer une expérience fascinante chez le spectateur. Devant un corps de guitare inversé sont tendues 3 cordes. Derrière les cordes est fixé un tambour de couleur sombre comprenant des lignes blanches horizontales. Lorsqu'on fait vibrer les cordes et tourner le tambour, l'œil perçoit les cordes comme des courbes ondulantes. Il s'agit d'une illusion optique : les lignes blanches décomposent le mouvement des cordes comme de la lumière stroboscopique. Les yeux humains ne voient dès lors que les points des cordes qui se trouvent devant les lignes blanches et recomposent ces images comme des lignes ondulantes.

Ned Kahn est en fait l'artiste représenté par le plus grand nombre d'installations, douze en tout, de la grande attraction « Tornade de feu » à des installations plus simples comme «Rain table». Ned Kahn est lui aussi passé par l'Exploratorium. Il reproduit des phénomènes naturels dans ses installations. Il utilise fréquemment l'organisation de la matière et l'intègre dans certaines installations qui montrent dès lors des phénomènes à l'esthétique fascinante.

« Secret Life » de Gregory Barsamian est une autre installation absolument fascinante qui, un peu à la manière de l'installation "Oscylinderscope » de Norman Tuck, utilise optique et mécanique pour créer une illusion optique renversante. Une grande sculpture à la forme d'un carrousel cylindrique tourne rapidement autour de son axe central. Le carrousel d'environ $3 \mathrm{~m}$ de diamètre et $2,5 \mathrm{~m}$ de hauteur comprend 16 barres verticales sur lesquelles sont fixées différents objets : œuf, main, hot dog, papier, rose, morceau de journal... Le carrousel est installé dans une vitrine sombre et est éclairé par lumière stroboscopique. Fréquence des flashs et rotation du carrousel sont synchronisées, de façon à ce que le spectateur ait lillusion que le carrousel ne tourne pas (c'est-à-dire que de prime abord, on ne perçoit pas la rotation du carrousel), les barres restent au même endroit. Le long de ces barres, les objets montent et

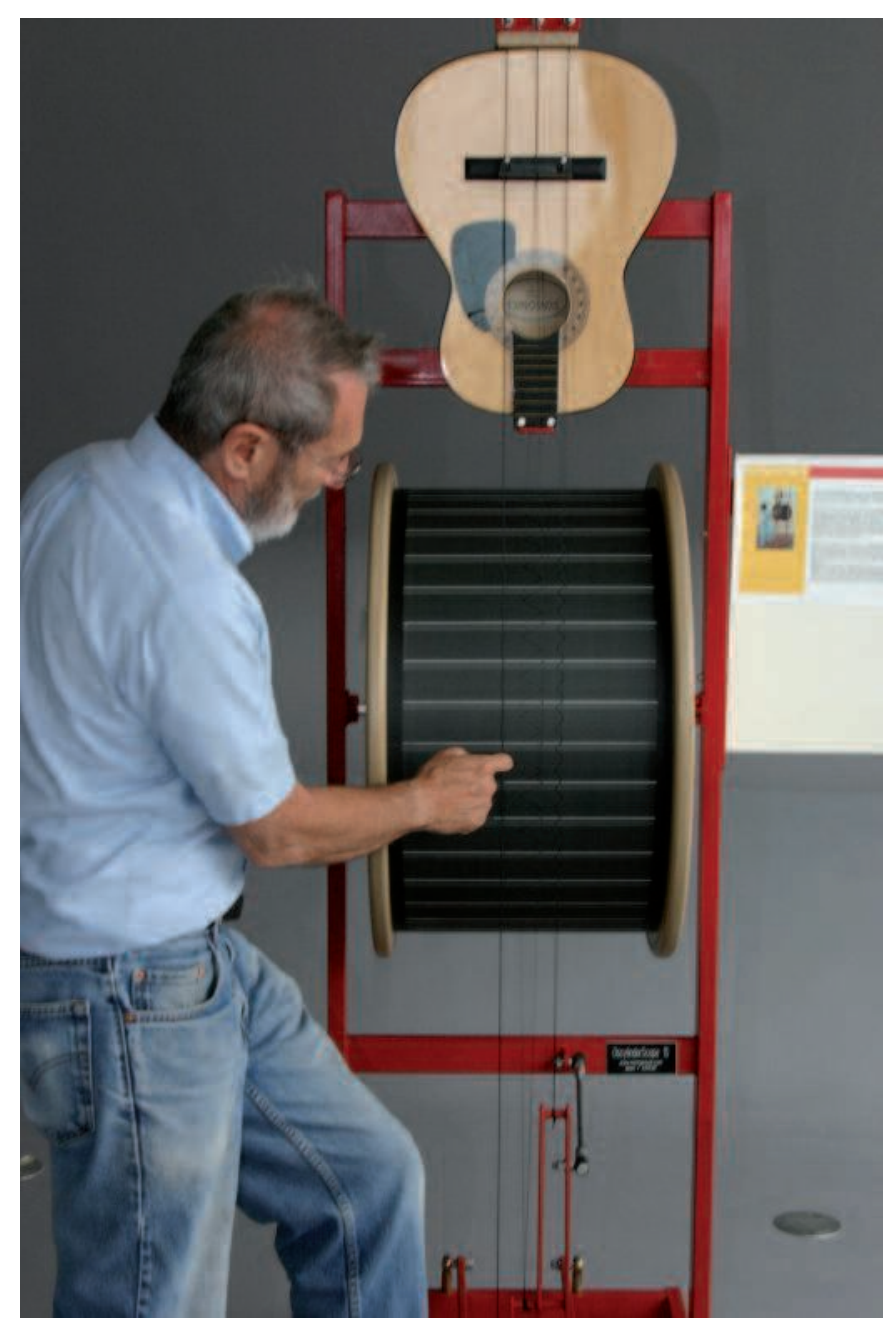

«Oscylinderscope » de et avec Norman Tuck (c) Phæno

descendent : une mini séquence surréaliste racontant la métamorphose d'un œuf d'où éclot une main qui saisit un hot dog puis en laisse tomber le papier d'emballage qui se transforme en une rose qui fane sur le sol... et ainsi de suite.

Directement au dessus de l'espace d'entrée du Phæno, « Ring ${ }^{3}$ » de Trimpin présente, suspendus, 3 anneaux aux diamètres de 3, 4 et 5 mètres l'un au dessus de l'autre. C'est en général la première installation que les visiteurs aperçoivent lors de leur arrivée. À l'intérieur de chaque anneau se trouve une sphère métallique qui a un mouvement de rotation le long de l'anneau. Les trois sphères tournent sans relâche, telles trois astres, plongeant nombre de visiteurs dans une contemplation méditative. Qui tente de comprendre le fonctionnement de l'installation, remarque que chaque anneau est 


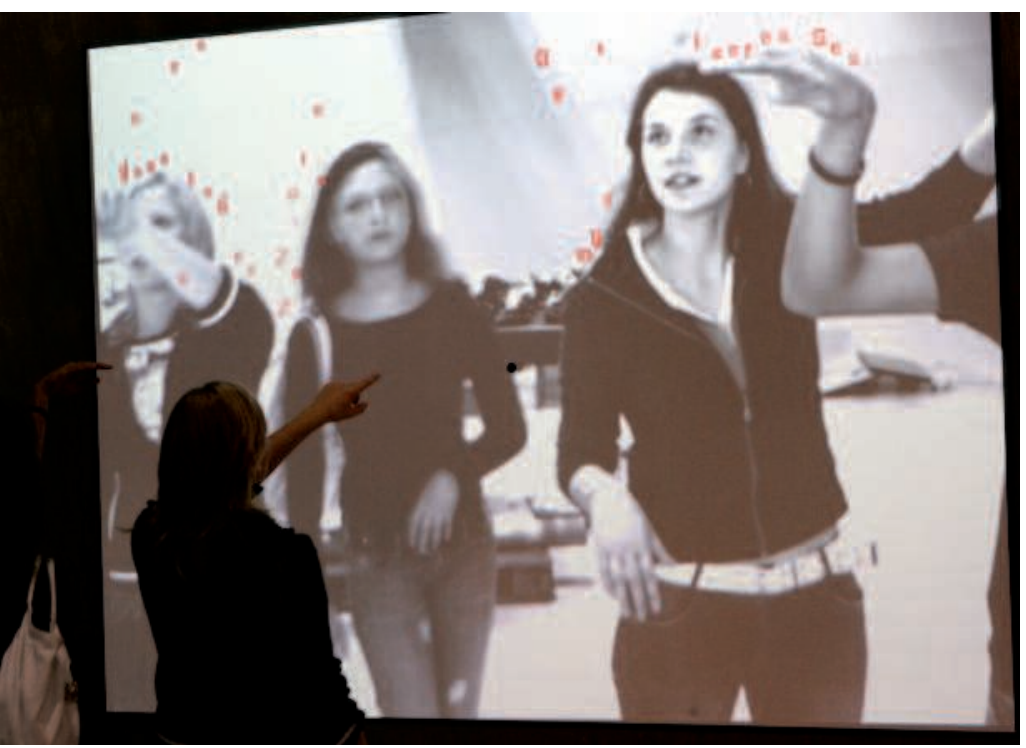

« Textrain » de Camille Utterback et Romy Achituv (c) Phæno

suspendu par quatre câbles entraînés par un système de vérins. C'est-à-dire que le mouvement des sphères est régulé de manière purement mécanique. Les vitesses de rotation des sphères sont variables, plusieurs modes sont programmés. Les chiffres 3, 4 et 5 et leurs rapports respectifs jouent un rôle primordial en mathématique (théorème de Pythagore) et en musique (harmonie). L'idée de "Ring3 » est venue à Trimpin, artiste sonore, en s'inspirant de la musique des sphères, une théorie développée au XVII ${ }^{e}$ siècle par Johannes Kepler.

L'exposition du Phæno comprend également des installations interactives d'art multimédia qui utilisent la vidéo pour plonger le spectateur dans la projection d'une animation virtuelle. Ainsi, dans "Textrain » de Camille Utterback et Romy Achituv, des lettres virtuelles tombent lentement sur une surface de projection blanche. Lorsque le visiteur se trouve devant cet écran, son image apparaît dans la projection. Si des lettres rencontrent un obstacle comme le bras, la tête, la jambe du spectateur, elles arrêtent leur descente et forment parfois des mots ou expressions ayant trait au temps météorologique ou au climat, d'où le nom de l'installation.

\section{Installations temporaires et développement futur}

À l'occasion de la Phænomenale 2007, un festival d'art et de science organisé - autour de la relation homme/machine - en collaboration avec la Kunstverein de Wolfsburg, Phæno a proposé deux installations de Robotlab («Autoportrait» et «Juke_bots »), un groupe d'artistes basés au ZKM (Zentrum für Kunst und Medientechnologie) de Karlsruhe qui réalise des installations utilisant des robots industriels tels qu'on peut en voir sur les lignes de production automobile par exemple et fait réaliser à ces robots des tâches typiquement humaines, voire artistiques, laissant parfois les robots interagir avec le public. Dans "Autoportrait », un robot devient artiste dessinateur alors que le public est le modèle. Le robot fixe à l'aide d'une caméra montée sur son bras l'image du visage d'un visiteur prenant la pose. Une fois l'image analysée par ordinateur, le robot dessine le portrait du visiteur ; son œuvre terminée, il la montre quelques secondes au public et efface l'image! Dans "Juke_bots ", ce sont deux robots qui jouent les DJ et font « scratcher » à tour de rôles des disques vinyles, produisant de la musique en direct.

Pour Phæno, l'objectif à moyen terme est de produire de nouvelles installations en collaborant avec des artistes, parfois locaux. Ainsi, l'édition 2008 de la Phænomenale qui aura pour thème "Choses en mouvement », permettra au public de découvrir une installation cinétique de Thomas Bartels, un artiste basé à Brunswick.

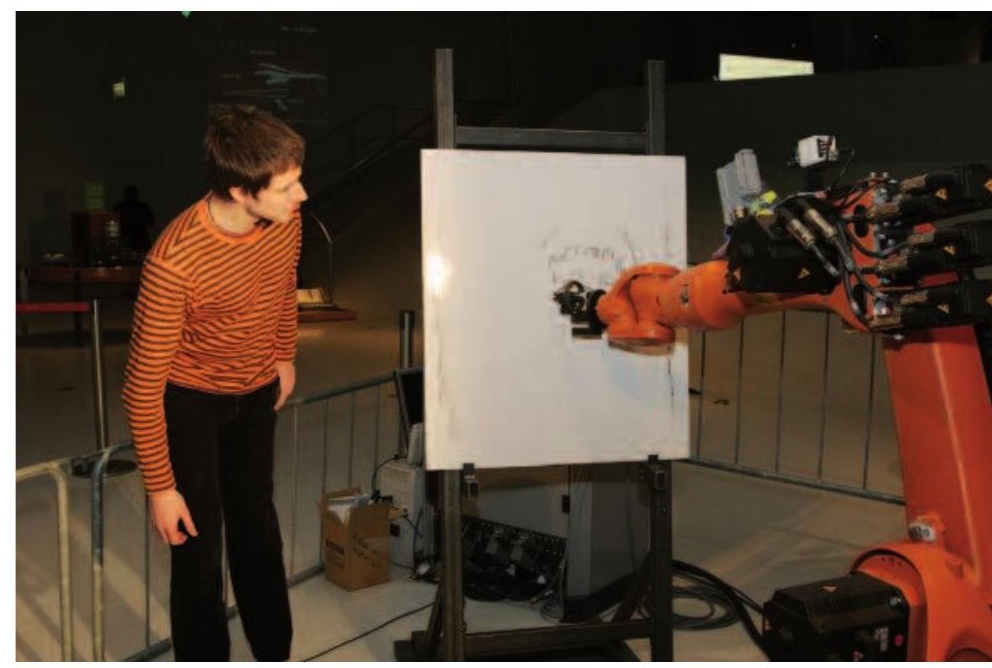

«Autoportrait » du groupe Robotlab présenté lors de la manifestation Phænomenale 2007. 
Depuis l'inauguration fin novembre 2005, environ 580000 visiteurs sont venus au Phæno et restent en moyenne 4 heures dans l'exposition. Ce résultat est synonyme de succès si l'on considère la particularité du site de Wolfsburg qui n’a pas la réputation d'être un site touristique. $44 \%$ des visiteurs sont des enfants et adolescents (jusqu'à 18 ans). $17 \%$ sont des groupes scolaires, $34 \%$ des familles. Environ $10 \%$ des visiteurs viennent d'une distance supérieure à 250 kilomètres ${ }^{(4)}$.

En définitive, Phæno apporte une nouvelle et importante contribution à l'amélioration de l'image de la ville de Wolfsburg, en rayonnant bien au-delà du Land de Basse-Saxe. Toutefois, le développement de Phæno et le succès qu'il rencontre auprès du public ne pourront être confortés que par des choix stratégiques visant par exemple à la constitution de son propre réseau d'artistes travaillant et créant autour de problématiques mêlant arts et sciences.

\section{Notes}

(1) Du coté français, citons l'article de Frédéric Edelmann « Zaha Hadid fait surgir le futur sur les terres de l'Allemagne automobile » paru dans Le Monde du 7 février 2006 et qui décrit le bâtiment en ces termes :

"L'animal, assez vif, doté d'un bec tranchant, a pourtant la douceur tranquille d'un dinosaure herbivore ». Enfin, la chaîne de télévision ARTE a produit et diffusé dans le cadre de la série « Architecture » un film documentaire de Richard Copans intitulé Phæno, le bâtiment paysage.

(2) Il fut décidé de mettre en place un jury d'experts issus de la scène des science centers pour sélectionner les stations d'expérimentation. Ce jury était composé de Remo Besio, directeur du Technorama en Suisse, Sir Richard Gregory, fondateur du premier science center en GrandeBretagne (Exploratory à Bristol, aujourd'hui @bristol), Peter Anderson, ancien directeur du Ontario Science Center et Joe Ansel.

(3) Parfois sur la base d'installations existantes. C'est par exemple le cas de « The little yellow chair » d'Arthur Ganson qui existait déjà dans une première version, mais a été radicalement transformée pour l'exposition du Phæno.

(4) Les statistiques données dans le texte datent du 26 février 2008. 